\title{
Techno-economic feasibility study of different WDM/TDM PON architectures
}

\author{
Bart Lannoo, Goutam Das, Maarten De Groote, Didier Colle, Mario Pickavet, Piet Demeester \\ Dep. of Information Technology, Ghent University - IBBT, Gaston Crommenlaan 8, B-9050 Gent, Belgium \\ Tel: (32) 9 14998, Fax: (32) 9 14899, e-mail: bart.lannoo@intec.ugent.be
}

\begin{abstract}
Introducing a WDM dimension on the top of a TDM PON system is a natural evolution to increase the capacity of the network, but this can also offer additional flexibility options. Different WDM/TDM PON flavours are compared to each other in terms of flexibility at the remote node and/or the ONU side. The considered architectures are then evaluated from a techno-economic point of view for a 10G technology, taking into account reasonable target costs for the optical components. In general the increased flexibility at the remote nodes has shown to have a limited influence on the cost, which opens good perspectives for more advanced WDM/TDM PON technologies. However, adding full flexibility in the optical layer at the ONU side will be too costly in the coming years, as the cost-per-user can be doubled or even more, compared to a fully "static" solution.
\end{abstract}

\section{INTRODUCTION}

Currently, telecom operators are adapting their networks for offering emerging services like high-definition video, video-conferencing, interactive gaming, etc. Optical fibre access networks can offer bandwidths that can therefore support an enormous variety of these services simultaneously. When considering the rollout of an optical access network, a choice must be made between two categories of fibre networks, either passive or active. Active optical networks (AONs) provide a (logical) point-to-point (P2P) connection between the central office (CO) and each user. Passive optical networks (PONs) on the other hand are point-to-multipoint (P2MP) networks, where the access fibre is shared by several users (e.g. 32, 64...), typically through a branched tree topology consisting of passive splitters between the $\mathrm{CO}$ and end-users.

PONs are an attractive access network solution thanks to their low operational costs and huge bandwidth. Today, the most used PON configuration is a (power splitting) time division multiplexing (TDM) passive optical network (PON), with gigabit-capable PON (GPON) and Ethernet PON (EPON) as the two most important standards [1-2]. However, these single channel systems are unable to provide sufficient bandwidth for all future services. The introduction of a wavelength division multiplexing (WDM) dimension on top of a TDM PON is the natural evolution to increase its capacity. In this way, WDM PONs and hybrid WDM/TDM PONs can be considered as strong candidates for next-generation PONs (NG-PONs).

In this paper we describe different hybrid WDM/TDM PON architectures and will compare them from a techno-economic perspective (based on capital expenditures, CAPEX). For our study, we consider a 10G PON technology and different ranges of flexibility, such as fully flexible dynamic bandwidth allocation (DBA) against partially flexible and even fixed wavelength allocation schemes. The techno-economic study includes an overview of the used network equipment (section 2) and the fibre installation (section 3). In this way, the equipment costs can be put in perspective of the fibre installation costs.

\section{HYBRID WDM/TDM PON ARCHITECTURES}

Hybrid WDM/TDM PON combines WDM and TDM technologies. The most common solution consists of several TDM PONs embedded in a WDM PON system. The advantage identified with respect to putting multiple TDM PONs in parallel is the possibility to manage in a flexible way the optical bandwidth. If the load on a specific wavelength is high, the demand for bandwidth on this wavelength can impose a critical load impacting all the services already connected. If there is a possibility to use a new wavelength operating at a low load, then this new wavelength will be preferred to limit the interruption of services on the previous wavelength. A general architecture of a (flexible) hybrid WDM/TDM PON is shown in Figure 1. In our model, three network parts are indicated between the optical line terminal (OLT) in the CO and the optical network unit (ONU) at the user side: feeder, distribution and last mile part.

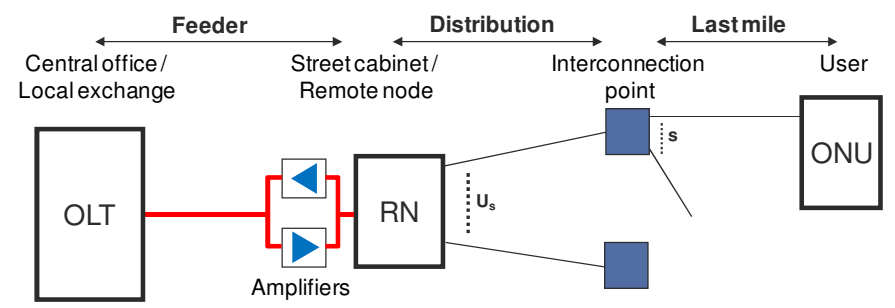

Figure 1: General model of the considered hybrid WDM/TDM PON architecture 
As OLT an architecture with $N_{u}$ uplink (UL) and $N_{d}$ downlink (DL) line cards is considered. The remote node $(\mathrm{RN})$, e.g. installed in a street cabinet (SC), and the ONU, will be varied in this paper. We assume there are $U_{s}$ TDM PON infrastructures and each TDM PON has $s$ ONUs. The power splitters of the TDM PONs are installed in so-called interconnection points (IcPs), e.g. in a SC or in the basement of a multi-dwelling unit (MDU).

For the ONU, two different types are discussed (Figure 2):

- ONU type a (ONUa) contains a tuneable burst mode transmitter (Tx) for UL data transmission and a tuneable optical filter for DL data transmission.

- ONU type $b(O N U b)$ contains a tuneable burst mode Tx for UL data transmission and two photo detectors (PDs), variable and fixed wavelength, for DL data transmission.

Both ONU types have a three-port circulator to separate UL and DL wavelengths. A WDM splitter can also be used for this purpose. The UL part has an UL line card and a tuneable burst mode Tx for tuning to any desired wavelength. The DL part has one DL line card, a classical PD and a tuneable optical filter for selecting the desired wavelength. The DL part of ONUb, however, has an additional PD, for receiving a fixed wavelength which is selected by using a fibre Bragg grating (FBG). The use of both a tuneable and fixed receiver (Rx) improves the DBA significantly and simplifies the corresponding MAC protocol [3]. When using only one tuneable Rx as in ONUa, the OLT has to store the wavelength each ONU was tuned to for the last frame. If an ONU is tuned to a wrong wavelength or it goes to sleep mode, however, the OLT has no means to detect the wavelength the ONU can listen to. Note that we do not intend to simultaneously use both wavelengths as then two (expensive) line cards per ONU are needed and we do not want to exceed the peak data rate of an individual ONU beyond $10 \mathrm{Gbps}$. However, as multiple ONUs are sharing the same TDM PON, they can tune to different wavelengths and hence create the possibility to increase the overall bandwidth usage of one TDM PON.
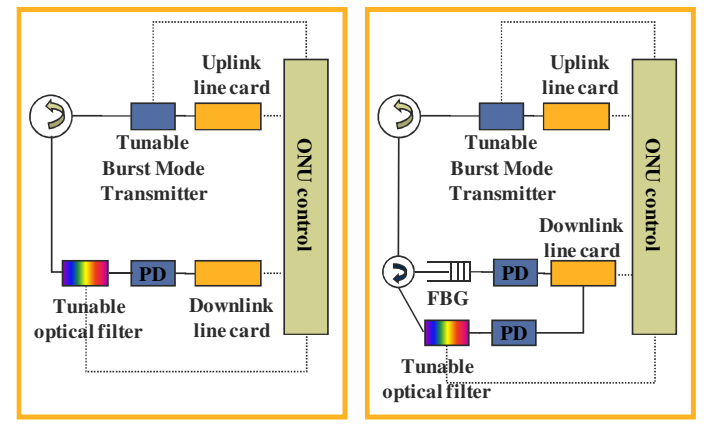

Figure 2: Architecture of ONU a (left) and ONU b (right)

For the RN, four types are considered (Figure 3):

- RN type 1 (RNI) is used for a power split PON (PS-PON), or broadcast \& select PON. The RN is a power splitter and all wavelengths are broadcast to all ONUs, which select the desired wavelength(s). This architecture is very simple and provides full flexibility, but it suffers from high power losses.

- RN type 2 (RN2) is used for a wavelength split PON (WS-PON). The RN is a wavelength splitter (e.g. AWG) and $U_{s}$ wavelength combiners are added to offer multiple wavelengths per TDM PON. Instead of transmitting all wavelengths to all TDM PONs, we provide $N_{d} / U_{s}$ DL wavelengths and $N_{u} / U_{s}$ UL wavelengths to each TDM PON. RN2 has a better power budget, but its flexibility is restricted.

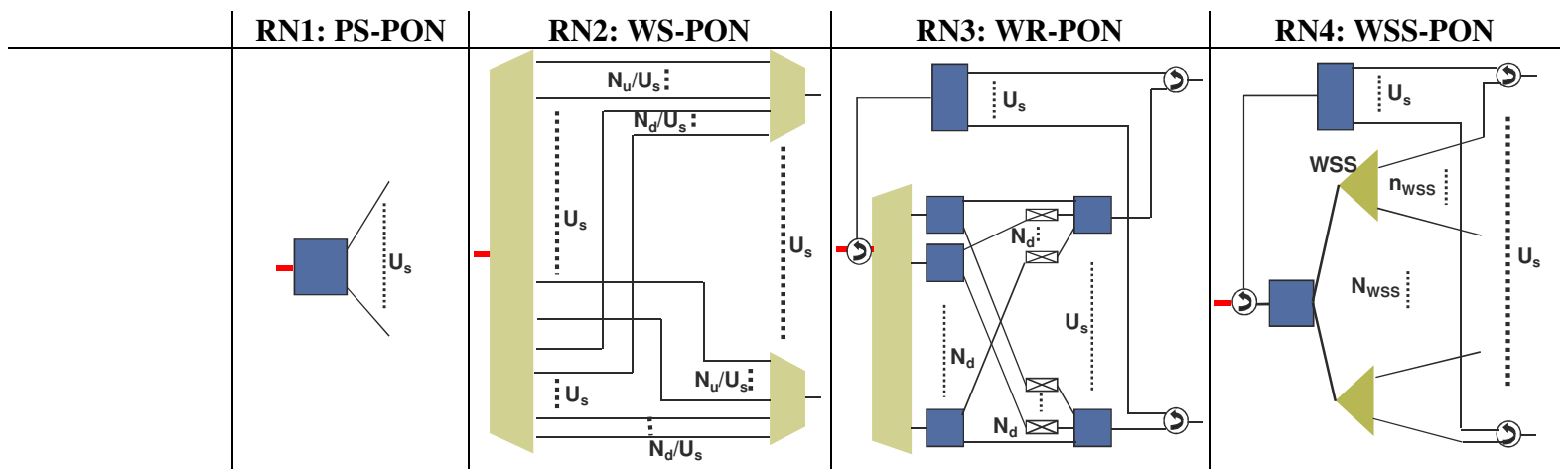

Figure 3: Overview of remote node $(R N)$ variants

- RN type $3(R N 3)$ is used for a wavelength routed PON (WR-PON). RN3 allows a fully flexible wavelength routing by adding some optical splitters/combiners and switches in the RN. It consists of a WDM splitter, a passive splitter stage, optical switches and a passive combiner stage. There are $N_{d}$ (if used for ONUa) or $N_{d^{-}}$ 1 (if used for ONUb) optical switches installed to control which wavelengths to be forwarded to which TDM PON. For the ONUb variant with $N_{d^{-}} 1$ optical switches (cf. Figure 3), each TDM PON has a fixed 
wavelength directly connected to it without being passed through an optical switch. For further details we refer to [3-4]. Note that the final power loss depends on the used switches.

- RN type 4 (RN4) is used for a wavelength selective switched PON (WSS-PON). By using some wavelength selective switches (WSS) [5], a partially flexible remote node is built. When a WSS is used as optical demultiplexer, it can steer each optical channel present on its common input port towards one of its output ports according to the wavelength of the channel. The power budget in the DL direction is much better than RN1. The RN4 variant shown in Figure 3 can only be used with ONUa. However, the architecture can be modified by adding an extra AWG and $N_{W S S} 2 \times 1$ combiners to be compatible with ONUb.

Two extreme cases in terms of flexibility and wavelength assignment are defined as benchmark architectures. In one extreme case (bench1), multiple separate TDM PONs are carried over different wavelengths, offering no flexibility. On the other extreme side (bench2), any wavelength can be delivered to any of the TDM PONs.

\section{MODEL FOR CALCULATING THE FIBRE INSTALLATION COSTS}

This section deals with the calculations of the analytical model, called simplified street length (SSL) model, used to estimate the magnitude of order of the installation (duct), cable and fibre lengths. In this model, the potential customer base is uniformly distributed over a squared area (Figure 4, left). One side of the square contains $n$ houses and the distance between two houses is indicated by $l$. The $\mathrm{CO}$ is always situated in the middle of the square. The model assumes that all houses can be connected in one line through the middle of the house (Figure 4 , right). All streets are connected using one divider street.

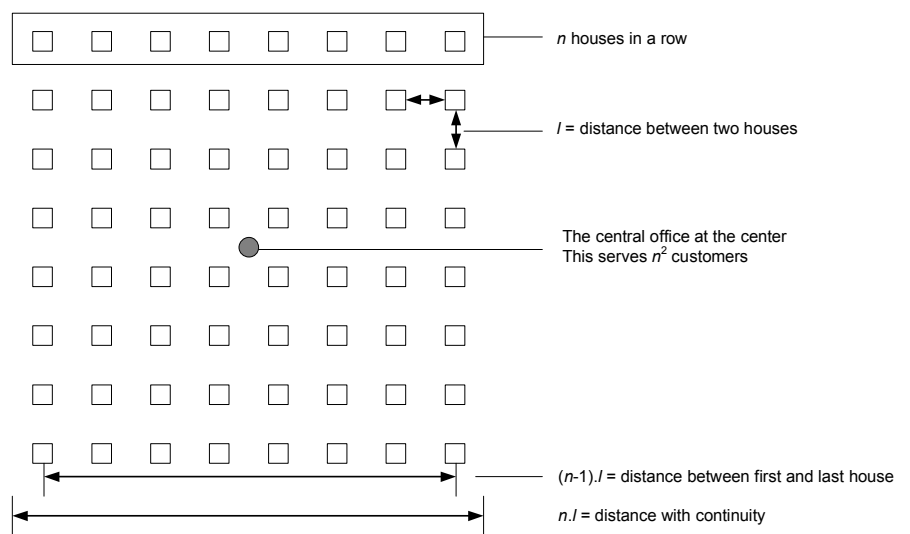

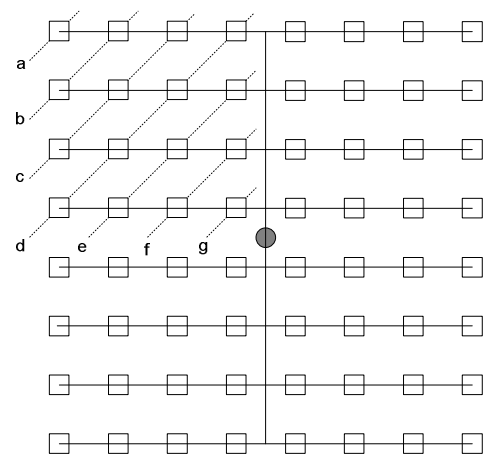

Simplified Manhattan street length model

Figure 4: Schematic overview of the parameters for the analytical installation and fibre length calculations

Regarding the installation length $I$, each row requires an installation length of $(n-1) . l$, and there are $n$ rows. The divider street requires an installation length of (n-1).l. Combined this gives an installation length as given in (1).

$$
I=n \cdot(n-1) \cdot l+(n-1) \cdot l=\left(n^{2}-1\right) \cdot l
$$

Regarding the fibre length $F$, the structure, as seen from the $\mathrm{CO}$ is fully symmetric and there are four equal quadrants. When we focus on one quadrant, we find for the houses in the categories indicated on Figure 4 (right) the following lengths: $a=(n-1) . l, b=(n-2) . l, \ldots g=l$. At the beginning, the number of houses per category is increasing with one each step. Once the diagonal line crosses at the half of the quadrant the number of houses per category is decreasing with one each step. The fibre length as such is given in (2).

$$
F=4 \cdot l \cdot \sum_{1}^{n-1}[\min (i, n-i) \cdot(n-i)]
$$

The general SSL model can be extended to a two-stage structure with two aggregation points, i.e. RN and IcP. For each network part (feeder, distribution, last mile), we can calculate the total fibre length between the endpoints (i.e. the number of fibres multiplexed in the RN and IcP, respectively), the cable length (where several fibres are aggregated together) and the installation length (where the duct is installed). With formula (1), we can calculate the total fibre length in each network part. The total cable length, however, has to be calculated in another way, since there is not always a separate cable between two endpoints. For the feeder and distribution part, the cable length is based on the fibre length, i.e. no sharing of cable between two different endpoints (i.e. between the $\mathrm{CO}$ and the RNs or between a RN and the IcPs). Multiple fibres between two identical endpoints share the same cable, as long as the number of fibres is not higher than the number of fibres per cable. The total cable length for the last mile part is based on the installation length for this concerned network part. It is assumed that if there is overlap in a certain part only one cable (or multiple cables based on the fibre granularity per cable) is needed in the last mile as we assume that the same cable passes different houses, and for each house one (or a 
few) fibre is terminated. Finally we have to calculate the duct length for each network part. If we consider a green-field situation, some ducts are used for more than one network part. To take this into account we first calculate the installation length of the feeder part. Further we calculate the installation length to connect all the IcPs (without taking into account the RNs) and subtract the feeder part to define the length of the distribution part. Finally we calculate the installation length to pass all the houses (without taking into account the RNs and IcPs) and subtract the distribution and feeder part to define the length of the last mile part.

\section{TECHNO-ECONOMIC EVALUATION}

For the calculations, two major cases are distinguished, an urban and rural case, respectively. The urban case considers an area with a population density of $5000 \mathrm{inh} . / \mathrm{km}^{2}$ (consisting of MDUs with 16 apartments) and a feeder fibre of at least $2 \mathrm{~km}$. The rural case considers an area with a population density of $300 \mathrm{inh} . / \mathrm{km}^{2}$ (consisting of single-family houses), and a feeder fibre of at least $10 \mathrm{~km}$. This rural case is most tricky in terms of infrastructure cost since the civil works (for installing the fibres) can have a large impact on the global cost.

Figure 5 shows a cost breakdown for the considered hybrid WDM/TDM PON flavours. Category 1 costs correspond to the civil works, category 2 costs to the transmission elements, and category 3 costs to the network elements. For the network elements (cat. 3), the costs of the key building components of the different architectures have been identified. However, extrapolations were mandatory to target acceptable values and in this way, we have assumed a distributed cost reduction to reach reasonable values within ca. five years (i.e. 2016). Adding full flexibility in the ONU (i.e. offering the possibility to receive all wavelengths in parallel, cf. bench2) seems yet unfeasible as it doubles the cost-per-user in urban areas, even with the cost targets for 2016. So, it is important to keep the ONU costs under control since this is a non-shared cost. On the other hand, adding some complexity in the RN and even in the ONU (cf. ONUb), already adds a substantial flexibility with a limited cost increase. This opens good perspectives for more advanced hybrid WDM/TDM PON technologies. For rural areas, however, the high costs for the last mile and distribution fibre installation, make it very difficult to define a valuable business case when a fully-buried green-field installation is considered.
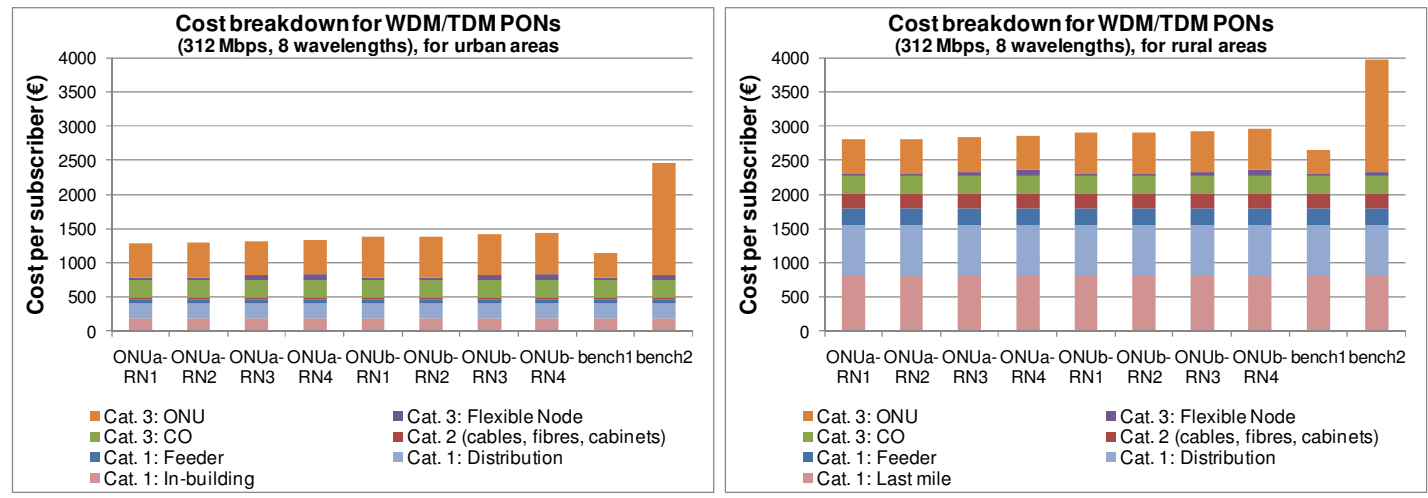

Figure 5: Cost breakdown for the cost per subscriber for different hybrid WDM/TDM PON technologies

\section{CONCLUSIONS}

Different hybrid WDM/TDM PON variants are discussed, and the main differences are indicated. Next to the equipment costs, the fibre installation costs are a very important cost factor. By using an analytical model, these installation costs are estimated for an urban and rural case, and put in perspective of the equipment costs. Note that especially for a rural case a fully-buried green-field installation is unfeasible. For the urban areas, however, the costs are reasonable, and some basic flexibility can be added without extremely affecting the final cost.

\section{ACKNOWLEDGEMENTS}

The research leading to these results has received funding from the EC in the FP7 ICT-ALPHA project.

\section{REFERENCES}

[1] IEEE 802.3ah task force home page [Online]. Available: http://www.ieee802.org/3/efm.

[2] ITU-T G.984.x series of recommendations [Online]. Available: http://www.itu.int/rec/T-REC-G/e.

[3] G. Das, et al: A New Architecture and MAC Protocol for Fully Flexible Hybrid WDM/TDM PON", in Proc. ECOC'09, P6.28, September 20-24, 2009.

[4] T. Koonen, et al: Flexibly Reconfigurable Fiber-Wireless Network using Wavelength Routing Techniques: The ACTS Project AC349 PRISMA, Photonic Network Comm., vol. 3 (3), pp. 297-306, July 2001.

[5] R. Jensen, A. Lord: Novel Non-Blocking Low Loss Scalable WSS Architecture, in Proc. OFC'08, OthA6, February 24-28, 2008. 